\section{MEDIO SIGLO DE VIVIENDA SOCIAL EN CUBA}

\author{
Dra. Arq. Dania González Couret ${ }^{1}$
}

\section{Resumen}

El próximo año 2010 cumple un siglo el primer barrio obrero proyectado y construido en Cuba. Después de 1959 la vivienda cubana se ha caracterizado por un estándar general y uniforme correspondiente al modelo de la vivienda social masiva nacida en Europa a inicios del siglo XX, y a diferencia de lo que sucede en otros países en desarrollo, continúa siendo una responsabilidad del Estado.

El artículo ofrece una panorámica de la evolución y tendencias de la vivienda social en Cuba durante la segunda mitad del siglo XX. Los inicios en los años '60s se caracterizaron por la búsqueda de tecnologías apropiadas y nuevas formas de expresión para la vivienda social masiva de un país en desarrollo y en Revolución, intentando

\section{HALF A CENTURY OF SOCIAL HOUSING IN CUBA}

Dra. Arq. Dania González Couret ${ }^{1}$

\section{Abstract}

2010 will be the 100th birthday of the first working-class neighbourhood planned and built in Cuba. Since 1959, Cuban housing is known for its general and uniform standards influenced by early XXth century European mass social housing. In contrast to the rest of developing countries, housing is still a responsibility of the State.

The present article offers an outlook of the evolution and trends of Cuban social housing throughout the second part of the XXth century. Its beginning, in the 60s, was characterized by the search for appropriate technologies and new ways of expression for the mass social housing of a developing and Revolutionary country. Also, the 
"erradicar" las condiciones habitacionales precarias, y las diferencias entre la ciudad y el campo. A partir de los '70s la vivienda cubana se vio comprometida con los sistemas de prefabricación de alta tecnología, como vía para dar solución a la demanda masiva. En los '80s cobró fuerza la conservación de los centros urbanos tradicionales y la crisis de los '90s obligó a abandonar definitivamente la industrialización pesada y buscar soluciones "alternativas". El trabajo concluye con el debate actual.

\section{PALABRAS CLAVE: VIVIENDA SOCIAL, HISTORIA DE LA VIVIENDA SOCIAL, EVOLUCIÓN DE LA VIVIENDA SOCIAL.}

Fecha de recepción: 16.06.2009

Fecha de aceptación: 04.11.2009

1 Cuba. Facultad de Arquitectura, Instituto Superior Politécnico José Antonio Echeverría, La Habana, Cuba,

Correo Electrónico: dania@arquitectura.cujae.edu.cu government tried to "eradicate" the precarious housing condition and the differences between urban and rural areas. From the 70s, in order to meet massive demands, Cuban housing centred its efforts on the high-tech prefabricated system. In the 80s, conservation of traditional urban centres was promoted and the crisis of the 90s forced Cuba to definitely quit heavy industrialization and search for "alternative" solutions. The current debate on the topic closes the article.

\section{KEY WORDS: SOCIAL HOUSING, SOCIAL HOUSING HISTORY, SOCIAL HOUSING EVOLUTION}

Received: 16.06 .2009

Accepted: 04.11.2009

1 Cuba. Facultad de Arquitectura, Instituto Superior Politécnico José Antonio Echeverría, La Habana, Cuba, Email:dania@arquitectura.cujae.edu.cu 


\section{Introducción}

El próximo año 2010 cumple un siglo el primer barrio obrero proyectado y construido en Cuba, específicamente en la ciudad de La Habana: El Barrio Pogolotti. A esta experiencia le sucedieron otras pocas, escasas y aisladas, durante la primera mitad del siglo XX, entre las cuales se destaca el llamado Barrio Obrero de Guanabacoa, donde ya en 1950 el Arq. Antonio Quintana proyectó, junto a la vivienda unifamiliar aislada, edificios multifamiliares que aun hoy exhiben su calidad de diseño y ejecución.

Después del proceso revolucionario iniciado en 1959, la vivienda promovida por el Estado se ha caracterizado por un estándar general y uniforme que en la mayoría de los casos se corresponde con el modelo de la vivienda social masiva nacida en Europa a inicios del siglo XX: viviendas totalmente terminadas "llave en mano", construidas con materiales duraderos (hormigón armado) a partir de proyectos repetitivos de edificios multifamiliares en urbanizaciones abiertas. Así, al contrario de lo que ha sucedido en otros países en desarrollo donde este modelo falló para la población de menores recursos, la vivienda social en Cuba continúa siendo una responsabilidad del Estado, aunque la población ha seguido produciendo viviendas unifamiliares por autoconstrucción.
A continuación se ofrece una panorámica de la evolución y tendencias de la vivienda social en Cuba durante la segunda mitad del siglo XX.

\section{Los inicios}

Al triunfo de la Revolución en 1959, la sociedad cubana estaba ampliamente estratificada y una buena parte de la población vivía en condiciones de pobreza. El problema de la vivienda había sido denunciado por Fidel Castro en su alegato de autodefensa durante el juicio por el asalto al Cuartel Moncada en $1953^{2}$, que se convirtió en el primer programa de acción de la Revolución triunfante ("El programa del Moncada"). Así, la vivienda constituyó uno de los objetivos más importantes entre los primeros programas sociales de la Revolución.

Las primeras acciones llevadas a cabo por la Revolución fueron encaminadas a mejorar las condiciones de vida de la población en áreas urbanas y rurales. Se "erradicaron" muchos asentamientos precarios y se entregaron nuevas viviendas a sus habitantes en comunidades o barrios diseñados y ejecutados integralmente con viviendas terminadas "llave en mano", servicios, infraestructura y espacios públicos. Las tipologías arquitectónicas

2 Castro, Fidel, 1953. 
empleadas fueron diversas, ya sea viviendas individuales aisladas y pareadas de una y dos plantas o edificios multifamiliares de tres y cuatro plantas. Las viviendas se construían generalmente con materiales y tecnologías tradicionales, pero también se emplearon elementos prefabricados de pequeño formato, como es el caso del sistema constructivo "Novoa", renombrado como "Sandino" a partir de la primera comunidad rural ejecutada con éste.

Estas primeras viviendas y urbanizaciones se caracterizaron por una alta calidad de diseño y ejecución, en correspondencia con las tendencias arquitectónicas más avanzadas de la época. En algunos casos, los proyectos arquitectónicos de los edificios de vivienda fueron repetidos en diversos lugares, pero existía una variada gama de diseños que, aunque en ocasiones se repetían, no llegaron a constituir proyectos típicos para reproducir a gran escala.

Desde los primeros años se inició la planificación física y territorial y se estructuró el sistema nacional de asentamientos. Las diferencias entre la ciudad y el campo eran notables, por lo cual se brindó especial atención al desarrollo de asentamientos rurales, con vistas a mejorar las condiciones de vida de la población en esas zonas y evitar, al mismo tiempo, la migración a la ciudad, garantizando la continuidad de la producción agrícola. Tanto en zonas rurales como urbanas se organizó y se inició la terminación del sistema educacional y de salud, con la ejecución de nuevas escuelas y centros asistenciales. Esto se complementó con obras e infraestructura para la producción industrial y agropecuaria y un sistema de instalaciones recreativas para la población, mediante las llamadas "playas del pueblo", en contraposición a las privadas existentes hasta entonces.

La realización más importante de esa época fue la "Unidad Vecinal Camilo Cienfuegos", en La Habana del Este, que siguió los parámetros del modelo urbano moderno (Figuras 1 y Foto 1). El conjunto de $28 \mathrm{Ha}^{3}$ quedaba rodeado por grandes avenidas al interior de las cuales el tránsito vehicular era sólo a partir de calles de servicio tipo "cul de sac". El espacio urbano, totalmente peatonal, quedaba conformado por edificios de diferentes diseños y alturas (tres tipos de edificios de cuatro plantas y dos tipos de proyectos de once pisos), e integralmente diseñado y ejecutado, con el equipamiento urbano y la delimitación del espacio público, semipúblico y privado, que tributaba a las viviendas en pantas bajas de los edificios de cuatro pisos. Los servicios se concentraron en una zona y los terrenos deportivos se ubicaron en el centro del conjunto, lo cual constituye una de las mayores críticas que ha recibido esta paradigmática urbanización. La ejecución fue financiada con los fondos de la lotería nacional y las viviendas resultaron muy económicas (60.00 USD por m2), a pesar de su alta calidad.

3 UIA, 1963, pág. 106. 
PLAN GENERAL DE LA UNIDAD VECINAL CAMILO CIENFUEGOS EN LA HABANA DEL ESTE. 1959 - 1961.

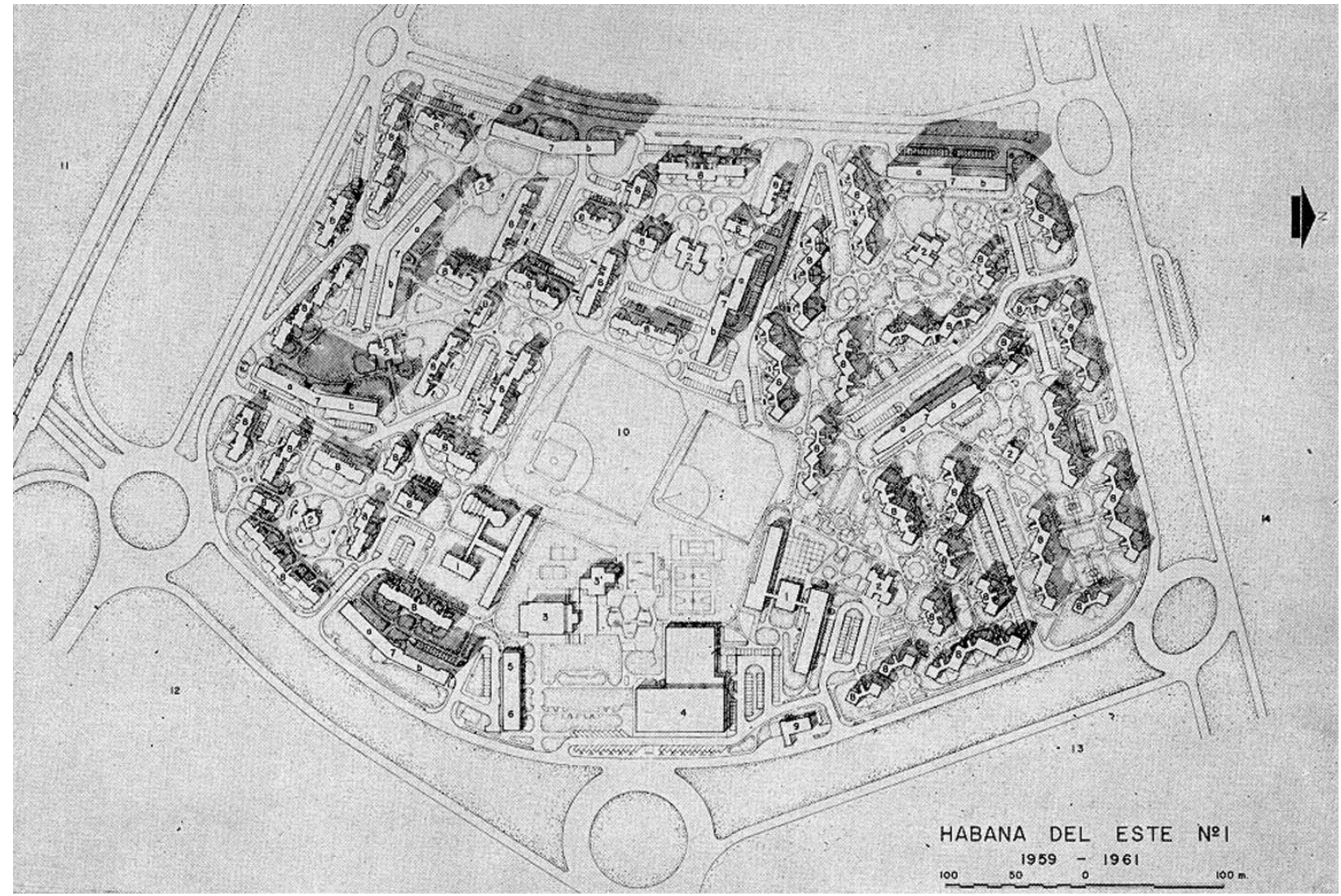

\section{FIGURA 1}

Fuente: Cuba. Architecture in Countries in the Process of Development. Seventh Congress if the International Union of Architects, La Habana. Septiembre de 1963. 


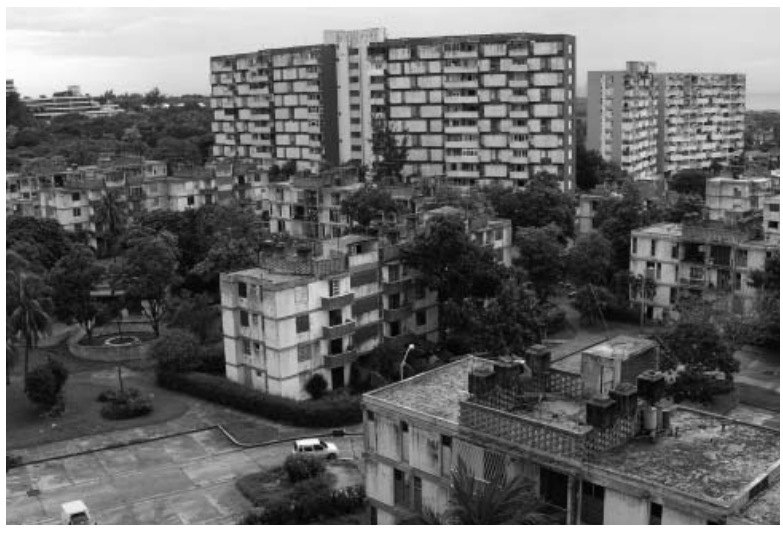

FOTO 1: Unidad Vecinal Camilo Cienfuegos en La Habana del Este. Vista actual de la urbanización. Foto de la autora, agosto 2008

Estas primeras realizaciones desarrolladas y financiadas por el estado no contaron con la participación de la población beneficiaria, ni en el proceso de concepción (planeamiento y diseño), ni durante la ejecución. En ocasiones, cuando las viviendas iban dirigidas a sectores poblacionales marginados y acostumbrados a vivir en condiciones precarias de subsistencia, tampoco se desarrolló una labor social y educativa que les permitiera asimilar las nuevas condiciones de vida que se le ofrecían y adaptarse a ellas, e ir transformando sus costumbres y formas de vida. El resultado fue que en ocasiones algunas familias abandonaron las nuevas viviendas e intentaron regresar a su lugar de origen y en la mayoría de los casos, las edificaciones se deterioraron rápidamente por el uso inadecuado y la falta de mantenimiento.

\section{Los '60s: en busca de la arquitectura social de un país en desarrollo y en revolución}

Numerosas fueron las experiencias desarrolladas en la primera década de la Revolución encaminadas a la búsqueda de tecnologías apropiadas y las necesarias formas de expresión de los nuevos "contenidos". Las investigaciones y la experimentación en relación con las tecnologías constructivas trataban de encontrar soluciones que permitieran construir rápidamente y de forma masiva viviendas económicas a partir de los recursos disponibles.

Se pueden identificar dos direcciones principales en estas búsquedas: por un lado, la experimentación con sistemas prefabricados de alta tecnología, y por otro, sistemas de elementos simples de pequeño formato, como es el caso del ya mencionado Sistema Sandino. Dentro de las soluciones de alta tecnología también pueden identificarse dos tendencias, ejemplificadas en sendos prototipos experimentales ejecutados, que intentaban mostrar caminos a seguir, aunque ninguno de los dos fue posteriormente repetido.

Una de estas tendencias proponía la producción de elementos prefabricados ligeros que permitieran armar células habitacionales espaciales para ser posteriormente izadas y situadas en su posi- 
ción definitiva dentro de cierta composición volumétrica. En el caso del proyecto de los arquitectos Mercedes Álvarez y Hugo D'acosta ${ }^{4}$, los elementos prefabricados ligeros eran de asbesto cemento, resistentes por su forma de doble curvatura e incorporaban de forma integral el equipamiento interior (Fotos 2 y 3). Esto daba como resultado un espacio mínimo como el de un tráiler, no susceptible de ser transformado. Por otra parte, los elementos delgados de asbesto cemento que por su poco peso favorecerían el izaje de las células habitacionales completas, nunca garantizarían condiciones térmicas apropiadas en los espacios interiores y su forma exterior generaría serios conflictos con la evacuación del agua de lluvias y la humedad.

Posteriormente, en los años '80s, los tres hijos de este matrimonio de arquitectos también abrazaron la profesión y en el trabajo de diploma realizado en un equipo conformado por los tres hermanos conducidos por sus padres, retomaron esta idea para el proyecto de una comunidad donde habitarían trabajadores de un centro de investigación, en el cual las unidades espaciales diseñadas por sus padres veinte años atrás se insertaban en una megaestructura que cruzaba por encima de la ciudad existente. Este proyecto, que hubiera encajado muy bien en las utopías de los años '60s, se encontraba en los '80s fuera de contexto, no sólo por el marco teórico de referencia a escala internacional, sino

4 Segre, Roberto, 1970. también por las condiciones materiales y económicas para su realización en Cuba.

Finalmente, el sueño de las unidades espaciales totalmente prefabricadas nacido en la Cuba de los años '60s, culminó en los '70s con la ejecución en el Centro Técnico para el Desarrollo de los Materiales de Construcción (antiguo Centro de Investigación de la Construcción), de un prototipo experimental de "célula habitacional" de hormigón armado que podría ser izada para el montaje de un edificio de viviendas al estilo del Hábitat 67 realizado por Moshe Safdie en Montreal.

El otro enfoque para resolver los problemas de la nueva vivienda en Cuba a partir del empleo de la alta tecnología fue promovido por el Arq. Fernando Salinas, quien fuera su principal ideólogo. Sus teorías acerca de la necesaria producción industrializada masiva y la economía de la vivienda, pero al mismo tiempo de su variedad, flexibilidad y adaptación al contexto así como a sus habitantes, constituyeron un paradigma, no sólo en Cuba, sino también en otros países de América Latina. A pesar de que la práctica cubana posterior no aplicó su teoría, ésta es aun hoy casi completamente válida ${ }^{5}$.

Esta tendencia abogaba también por unidades modulares, pero que en este caso no constituían células habitacionales completas, sino módulos estructurales abiertos y flexibles para la transformación del

5 González, Dania, 2002, pags. 32 - 35. 


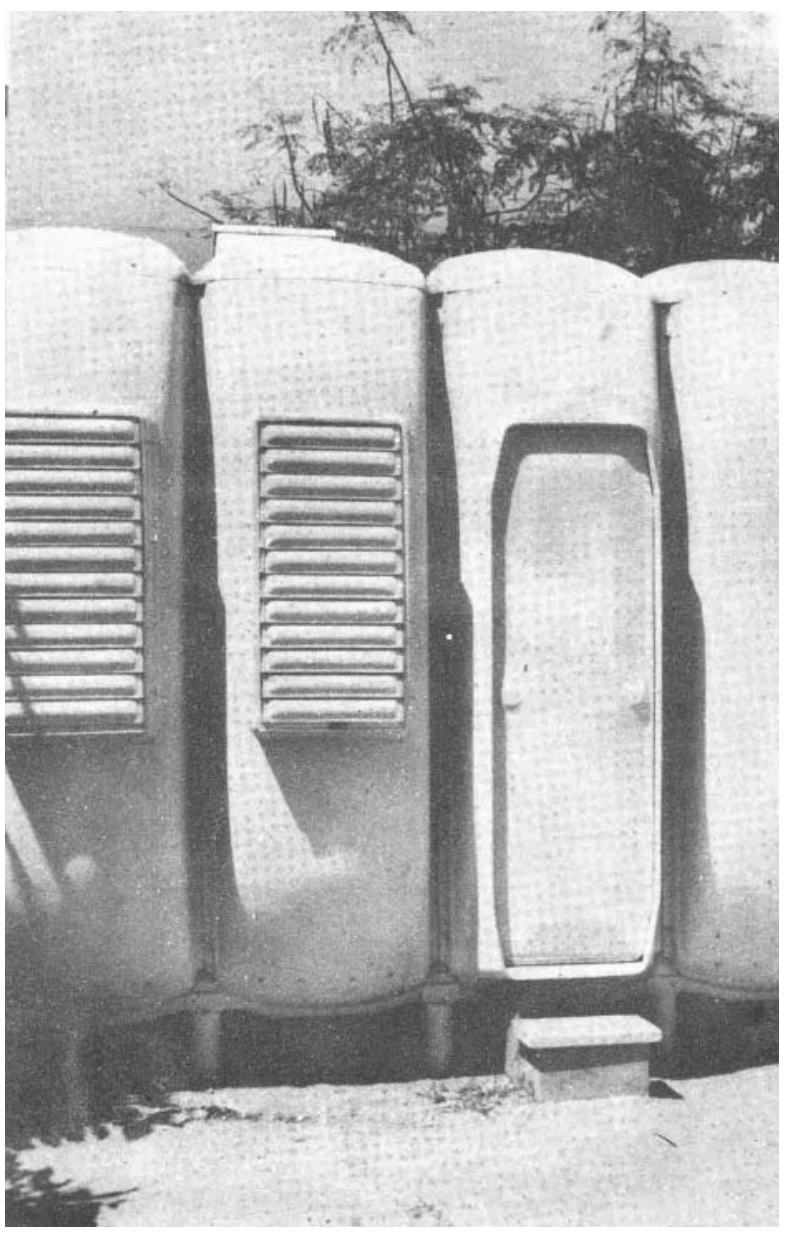

FOTO 2: Vivienda experimental en materiales laminares. Mercedes Álvarez y Hugo D’acosta, 1965 - 1968. Imagen exterior del prototipo realizado en asbesto cemento. Fuente: SEGRE, Roberto. 10 años de Arquitectura en Cuba Revolucionaria. $1^{a}$. ed. La Habana, Ediciones Revolucionarias. 1970.

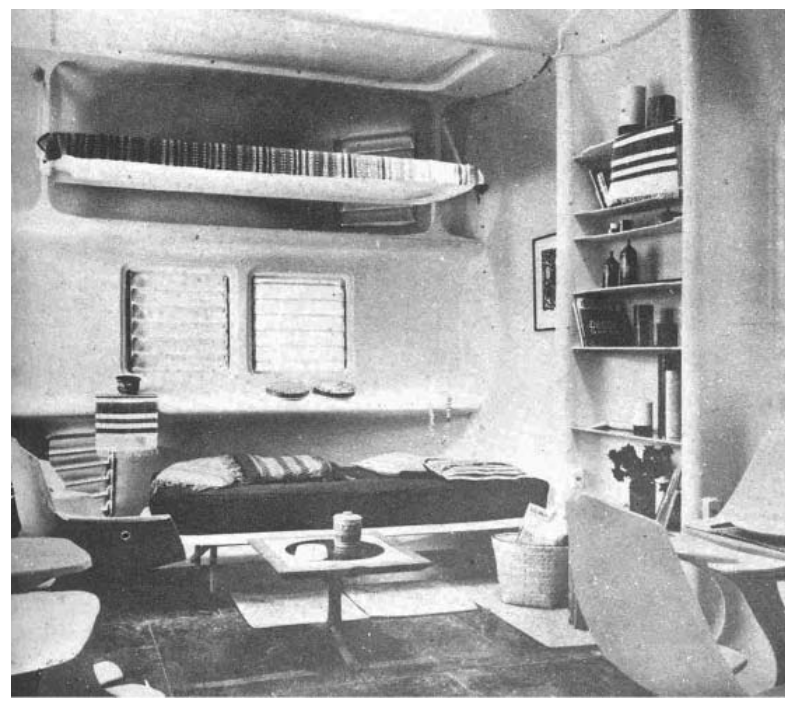

FOTO 3: Vivienda experimental en materiales laminares. Mercedes Álvarez y Hugo D'acosta, 1965 - 1968. Imagen interior del prototipo realizado en asbesto cemento. Fuente: SEGRE, Roberto. 10 años de Arquitectura en Cuba Revolucionaria. $1^{\text {a }}$. ed. La Habana, Ediciones Revolucionarias. 1970. 


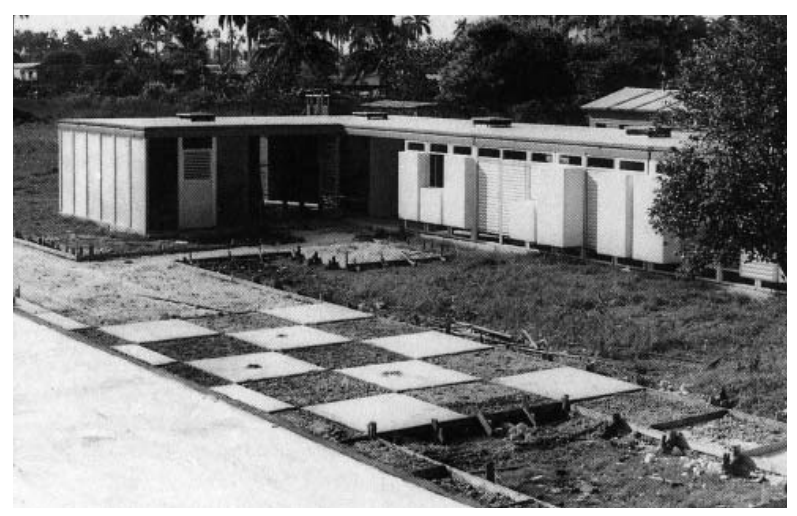

FOTO 4: Prototipo experimental de vivienda construida con el sistema Multiflex, 1969. Imagen exterior. Foto de Paolo Gasparini. Fuente: VEJAR, Carlos. Y el perro ladra y la luna enfría. Fernando Salinas: diseño, ambiente y esperanza 1a . ed. La Habana, Ediciones Unión. 1994.

espacio interior, subdividido mediante elementos ligeros y el propio equipamiento, y con cierres prefabricados, también modulares, que permitían una variedad en la expresión exterior de las viviendas, siempre y cuando se tratara de un conjunto pequeño que no llegara a resultar monótono. Es el caso del sistema abierto "Multiflex" (Fotos 4 y 5), que venía siendo elaborado por Salinas desde 1965 y fue aplicado en un trabajo presentado por un equipo de estudiantes conducido por él y premiado en el concurso estudiantil del Congreso Internacional de la Unión Internacional de Arquitectos de 1969 en Buenos Aires ${ }^{6}$. Sólo un prototipo experimental

Vejar, Carlos, 1994.

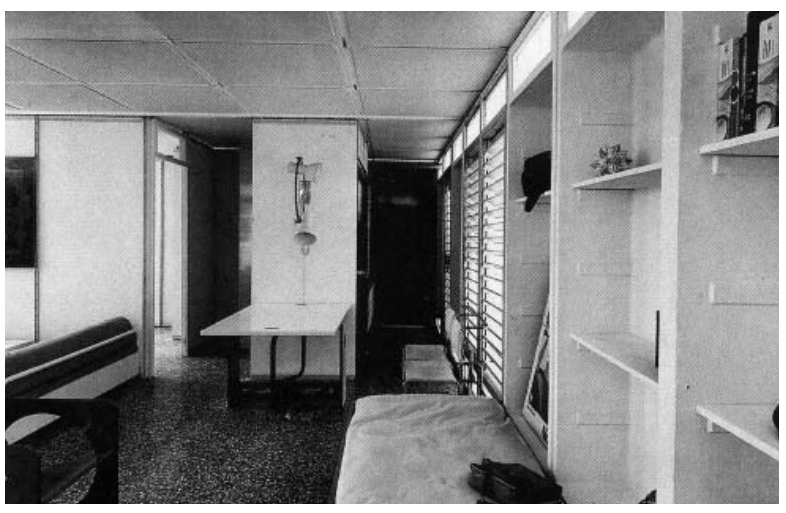

FOTO 5: Prototipo experimental de vivienda construida con el sistema Multiflex, 1969. Imagen interior. Fuente: VEJAR, Carlos. Y el perro ladra y la luna enfría. Fernando Salinas: diseño, ambiente y esperanza 1a . ed. La Habana, Ediciones Unión. 1994.

de este sistema integrado por varios módulos fue construido en El Wajay, en la periferia de la ciudad de La Habana. Posteriormente, otras pocas realizaciones, todas experimentales, han retomado este concepto de la vivienda "soporte", pero finalmente, las divisiones interiores ligeras y móviles han debido ser sustituidas por elementos sólidos fijos, ante la imposibilidad de ser mantenidos y repuestos por parte de sus habitantes.

A pesar de la limitada aplicación de estos planteamientos teóricos, la vivienda social cubana de los ‘60s fue, en general, altamente cualificada. Los edi- 
ficios multifamiliares hasta tres o cuatro plantas siguieron los códigos de la arquitectura moderna con influencias brutalistas, pero teniendo en cuenta las condiciones climáticas calido - húmedas, y las soluciones espaciales interiores trataron de ser tan flexibles como las tradicionales paredes de carga lo permitían. A pesar de la repetitividad de los proyectos, la extensión de los conjuntos desarrollados fue limitada a los espacios ya urbanizados pero no edificados dentro de la trama urbana de la ciudad consolidada, de manera que se pudo evitar la monotonía que la repetición a gran escala hubiera podido generar. Por otra parte, se completaba la urbanización con los espacios públicos, el equipamiento, la vegetación, y tanto los materiales de construcción y terminación como la ejecución eran de buena calidad.

\section{El compromiso con la prefabricación pesada interrumpió la continuidad de las experiencias anteriores.}

La planta de prefabricación de "grandes paneles" donada por la Unión Soviética a finales de los "60s ubicada en San Pedrito en la ciudad de Santiago de Cuba, ocasionó importantes cambios en la construcción de viviendas en Cuba, ya que a partir de entonces fue necesario construir masivamente viviendas que usaran esa tecnología. La primera ex- periencia de aplicación de estos sistemas fue en el Microdistrito José Martí de esa ciudad, donde los profesionales cubanos adecuaron los moldes para elementos de pared (de $30 \mathrm{~cm}$ de espesor por las necesidades de aislamiento en el clima frío de la URSS) a las condiciones cubanas, tanto climáticas como económicas. Esto condicionó la expresión del único proyecto arquitectónico típico de edificio en forma de un bloque prismático con el que se conformó esta urbanización (Foto 6).

Posteriormente, se desarrollaron los sistemas cubanos de grandes paneles y se instalaron diversas plantas de prefabricación para la producción de los elementos de estos sistemas en diferentes lugares del país. El llamado Gran Panel 4, más que un sistema constructivo, era un proyecto típico de un edificio prismático de cuatro plantas, que había sido descompuesto en partes (paredes, entrepisos y cubiertas), para ser prefabricadas por separado, pero que cuando éstas eran izadas y "montadas" en el lugar que definitivamente ocuparían durante toda la vida útil del edificio, el resultado era siempre el mismo.

Este único proyecto se repitió en todas las llamadas "zonas de nuevo desarrollo" que surgieron en la periferia de las ciudades cubanas, durante los años '70s y '80s. En la ciudad de La Habana, por el contrario, la mayoría de los edificios construidos en estas urbanizaciones abiertas al estilo del Movimiento Moderno, no eran precisamente prefabri- 


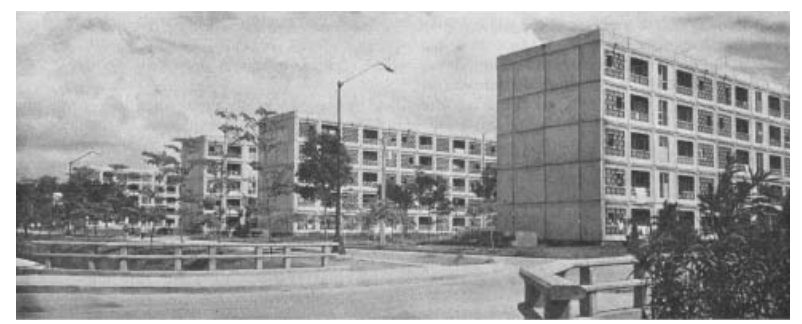

FOTO 6: Distrito José Martí en Santiago de Cuba, 1970. Fuente: REBELLÓN, Josefina. Arquitectura y desarrollo nacional. Cuba 1978. 1ª. ed. La Habana, Editorial CEDITEC. 1978.

cados, sino "semi" prefabricados (se prefabricaban en obra los entrepisos y cubiertas, pero las paredes eran de albañilería tradicional), a pesar de lo cual, también se repitió casi un único proyecto típico.

Este proyecto típico que se extendió por todo el país, ni siquiera se adecuaba a los requerimientos del clima cálido - húmedo de Cuba, ya que era construido con paneles delgados $(10 \mathrm{~cm})$ de hormigón armado sin protección contra el sol y la lluvia. Por otra parte, no se adecuaba a las tradiciones ni a la identidad local. Así, al salir de cada centro urbano o poblado, perfectamente identificable y adentrarse en los barrios de nuevo desarrollo, resultaba imposible adivinar en qué lugar del país el observador podría encontrarse. Sin embargo, el

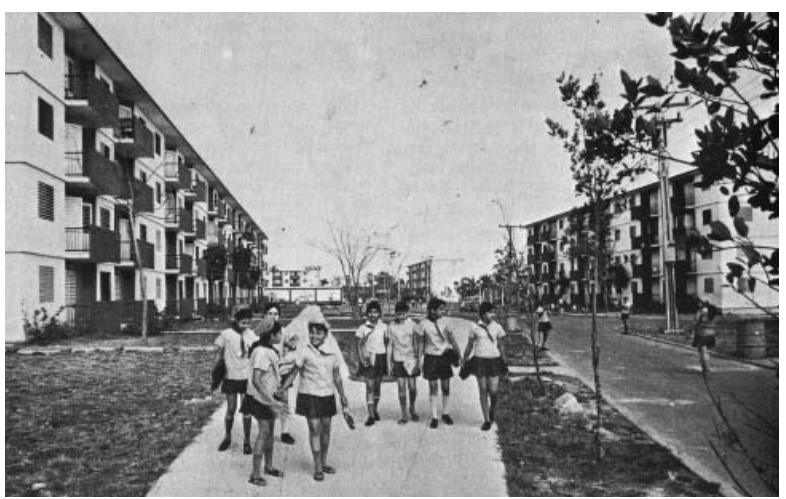

FOTO 7: Comunidad rural pecuaria en La Habana, década de los años 70's. Fuente: REBELLÓN, Josefina. Arquitectura y desarrollo nacional. Cuba 1978. 1ª ed. La Habana, Editorial CEDITEC. 1978.

uso de estos sistemas y proyectos típicos permitió construir viviendas de forma rápida y masiva en la década de los años '70s.

Lamentablemente, en ocasiones estos proyectos fueron también construidos en asentamientos rurales, con la intención de mejorar las condiciones de vida de los campesinos (Foto 7). Entonces, las ideas de la "modernidad" y la buena calidad de las viviendas se asociaban al edificio multifamiliar de hormigón armado, mientras que la vivienda rural tradicional (el bohío) realizado con materiales naturales disponibles localmente y de excelente comportamiento térmico se asociaba con atraso y pobreza. Así, en los asentamientos rurales donde se introdujo el edificio multifamiliar como símbo- 
lo de modernidad y bienestar, se combinaron las desventajas de la vida urbana (falta de privacidad y relación con el suelo para la producción de alimentos) con las de la vida rural (lejanía y bajo nivel de servicios y comunicación), lo cual unido al carácter cerrado de estos asentamientos donde los descendientes no podían producir su propia vivienda para continuar habitando en el lugar, fue una de las causas del envejecimiento sin reemplazo de estas poblaciones, el abandono de las mismas por las nuevas generaciones, y por tanto, la falta de mano de obra agrícola que se produjo en las siguientes décadas ${ }^{7}$.

Un fenómeno que caracterizó la producción de viviendas en Cuba durante los años '70s fue el Movimiento de Microbrigadas, a través del cual los centros de trabajo constituían brigadas para construir viviendas que una vez terminadas, eran otorgadas en asambleas de trabajadores a aquellos que más las necesitaban o las merecían, con independencia de que hubieran participado o no en su ejecución. El trabajo de los que iban a construir las viviendas era asimilado por el resto del colectivo, de manera que todos con su esfuerzo contribuían al logro de ese objetivo. Por esta vía se construían, fundamentalmente, edificios semi prefabricados, con tecnologías tradicionales, que no requerían de mano de obra altamente calificada. La población (no necesariamente los futuros habitantes de las viviendas) participaba en la construcción de los edificios, pero no en su planeamiento y diseño.

Además de los sistemas prefabricados de Grandes Paneles, otros sistemas constructivos de alta tecnología fueron desarrollados durante los años '70s por Centro Técnico de la Vivienda y el Urbanismo, que contaba con una planta experimental de prefabricado donde se produjeron todos los elementos con los cuales fueron construidos los edificios altos del Microdistrito Plaza de la Revolución en el corazón de La Habana (Foto 8), y que fueron posteriormente exportados al resto de las ciudades del país. Este microdistrito fue desarrollado en una zona que ya se encontraba urbanizada pero no edificada en 1959, a partir de una trama en retícula que fue parcialmente alterada por la introducción de edificios del tipo pantalla y superbloques. No obstante, el ordenamiento urbano tradicional y su centralidad han sido factores positivos que diferencian este microdistrito de otros localizados en la propia ciudad de La Habana o en otras ciudades del país.

Entre los sistemas constructivos de alta tecnología experimentados en esta zona de la ciudad de La Habana y extendidos posteriormente al resto del país se encuentra el sistema yugoslavo IMS (Instituto de Materiales de Servia), compuesto por tramos de columnas prefabricadas con una longitud total de cuatro pisos y losas casetonadas también

7 González, Dania, 1993, pags. 51 - 60. 
prefabricadas en plantas, que se unían por medio de cables postesados (Foto 9). Otro ejemplo lo constituye el sistema constructivo de Moldes Deslizantes, desarrollado con asesoría húngara a partir del empleo de hormigones de altas prestaciones, de manera que los moldes y toda la superficie de trabajo se iban desplazando hacia arriba mediante la acción de mecanismos hidráulicos, para dejar conformados los muros de hormigón armado (Foto 10).

Con estos sistemas constructivos se desarrollaron varios proyectos que fueron posteriormente adaptados y construidos en otras ciudades cubanas, pero las soluciones arquitectónicas no se adecuan a los requerimientos climáticos (ventanas de vidrio expuestas sin protección solar) ni espaciales (ausencia de espacios para lavar y secar la ropa) ni urbanos, pues su inserción en centros urbanos como el de Pinar del Río y Ciego de Ávila (Foto 11) generó un alto contraste y ruptura con la ciudad tradicional.

Los primeros intentos de rehabilitación urbana durante los años '70s ignoraron los valores de la ciudad tradicional. El paradigma del Movimiento Moderno estaba vivo aún y manzanas tradicionales completas fueron demolidas para insertar nuevos edificios típicos de viviendas sin tener en cuenta las regulaciones urbanas vigentes. Un buen ejemplo de esto lo constituyen las intervenciones urbanas desarrolladas a finales de la década en el barrio de Cayo Hueso en Centro Habana y en la Esquina de Tejas en El Cerro.

\section{Recuperando la ciudad tradicional}

El reconocimiento de la superioridad de las zonas urbanas tradicionales con respecto a las abiertas de nuevo desarrollo reconocido a escala internacional, así como la declaración de La Habana Vieja como "Patrimonio de la Humanidad" en 1982, impulsaron el cuestionamiento del modelo urbano moderno, el estudio de la ciudad tradicional para encontrar nuevas y mejores soluciones adaptadas a las condiciones contemporáneas ${ }^{8}$, y con ello el proceso de rehabilitación urbana cobró fuerza.

Se inició así el trabajo de rehabilitación del centro histórico de La Habana, que constituye hoy un ejemplo paradigmático a escala internacional de un proceso de gestión que permite recuperar el patrimonio, autofinanciarse con los ingresos provenientes del turismo $y$, a la vez, mantener a los tradicionales residentes y, conservar, por tanto, no sólo el ambiente urbano, sino el ambiente social. La rehabilitación de viviendas en áreas urbanas centrales presenta una particularidad importan-

8 Alfonso, Díaz y De la Peña, 1989, pags. 2 - 7. 
te en Cuba: las personas que originalmente viven en el lugar toman parte en el proceso y continúan viviendo en el sitio después que la rehabilitación concluye, a diferencia de lo que sucede en la mayoría de los países, donde una vez que una zona central es rehabilitada, los originales habitantes del área degradada se ven forzados a abandonarla por los altos precios del ambiente recalificado.

Asociado al fenómeno de la rehabilitación urbana se crearon en los años '80s los "Talleres Integrales para la Transformación del Barrio", para ayudar a los habitantes de las zonas urbanas más deterioradas de la ciudad de La Habana en el mejoramiento de sus condiciones de vida y de la calidad ambiental en esas áreas degradadas. La rehabilitación de ciudadelas $^{9}$ fue y continúa siendo aun hoy, otro importante objetivo en la conservación de viviendas y la elevación de sus condiciones de habitabilidad.

El proceso de rehabilitación urbana ha originado en ocasiones un nuevo tipo de vivienda temporal o "de tránsito" hacia donde se trasladan de forma no definitiva los habitantes cuando las acciones constructivas a desarrollar en los edificios así lo requieran. Sin embargo, la Dirección de Planificación Física de la ciudad de La Habana ha demostrado que la ciudad tiene potencialidades para crecer sobre sí misma durante al menos veinte años, manteniendo la población tradicional, si se desarrolla un proceso de "rehabilitación integral" que tome la manzana como unidad básica de planeamiento.

No sólo consideraciones teóricas, sino también razones prácticas han motivado e impulsado la recuperación de la ciudad existente. Durante la segunda mitad de la década de los años '80s se revitalizó el Movimiento de Microbrigadas, pero en este caso no para continuar construyendo edificios típicos de vivienda en zonas abiertas de nuevo desarrollo, sino proyectos de edificios atípicos en lotes disponibles dentro de la trama consolidada de la ciudad, lo cual contribuiría a reducir el crecimiento urbano (Foto 12).

A pesar de que se suponía que estos proyectos eran específicos para adecuarse al contexto, algunos de ellos se repitieron varias veces en diferentes partes de la ciudad en aras de ahorrar tiempo en la labor de diseño. Los arquitectos tuvieron la posibilidad de desarrollar diseños sin las restricciones impuestas por la prefabricación y la estandarización, sin embargo, el modelo seguido en muchos casos se corresponde en lo formal con el edificio de viviendas de los años '50s, pero con una calidad espacial y ambiental interior inferior. Los arquitectos no estaban acostumbrados a trabajar en esas condiciones

9 Se refiere a una tipología habitacional mínima donde cada familia habita prácticamente en un solo espacio, con servicios comunes. Puede tratarse de edificaciones construidas así con fines especulativos u otras que se han ido subdividiendo. 


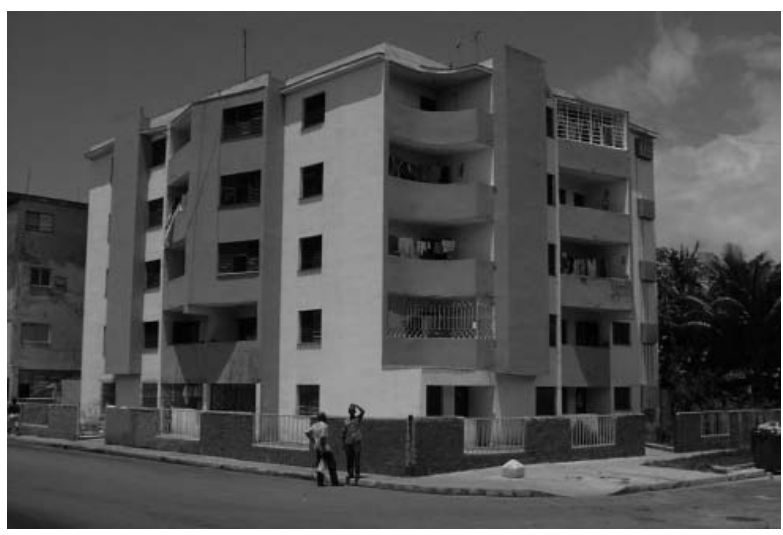

FOTO 12: Proyecto de edificio de viviendas "atípico" construido por las Microbrigadas en El Vedado, en la década de los años '80s. Foto tomada por la autora, 2000.

\section{PLAN GENERAL DE LAS ARBOLEDAS, DÉCADA DE LOS AÑOS '80S.}

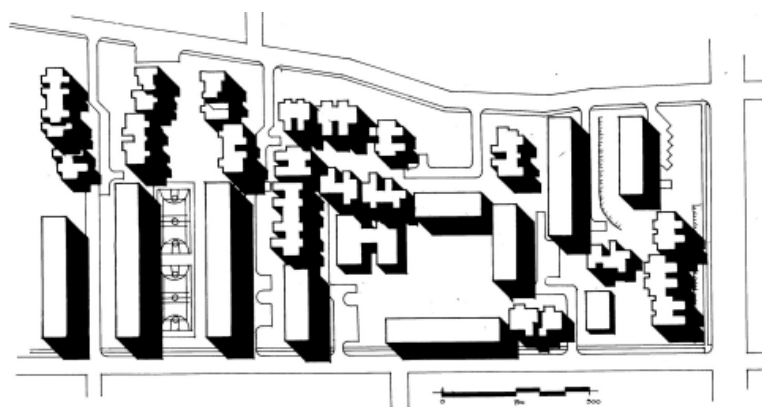

FIGURA 2

Fuente: ARMAS, Rendel, y MORALES, Boris. Estudio de modelos urbanos para zonas residenciales en Ciudad de La Habana. Trabajo de Diploma, Facultad de Arquitectura, ISPJAE. La Habana. 2000. Tutor: Dra. Arq. Dania González.

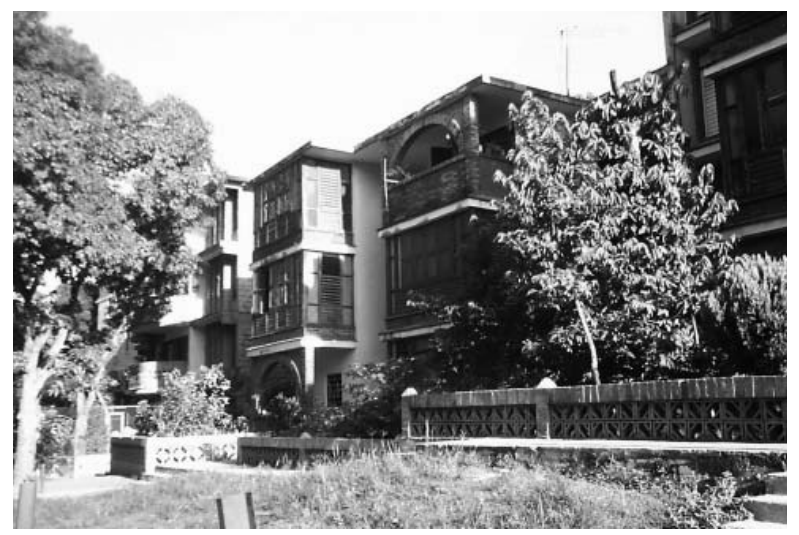

FOTO 13: Las Arboledas. Foto tomada por la autora, 2000.

de libertad ni a diseñar dentro de la trama urbana tradicional y no realizaron un adecuado estudio del repertorio anterior para retomar las experiencias más positivas. Por el contrario, se limitaron en la mayoría de los casos a imitar miméticamente el diseño exterior de los edificios circundantes construidos 30 años atrás en una falsa integración contextual y se perdió así la posibilidad de enriquecer la ciudad, contribuyendo con la imagen de la nueva arquitectura de la vivienda social cubana contemporánea. En otros casos, por el contrario, los arquitectos siguieron las tendencias internacionales sin adecuarlas a las condiciones y necesidades locales.

Con independencia del inicio del proceso de rehabilitación que caracterizó la década de los '80s, durante estos años también se desarrollaron otras 
experiencias urbanas de interés en la ciudad, que marcaron un cambio importante con respecto a lo que se venía haciendo hacia ese momento. El proyecto de "Las Arboledas" (Figura 2 y Foto 13) fue comenzado a finales de los '70s por un equipo liderado por el arquitecto norteamericano Hook Rorick en colaboración con la entonces Empresa de Diseño de Viviendas del gobierno de la ciudad, hoy denominada "Diseño Ciudad Habana" (DCH).

La solución de diseño urbano se adecuó al contexto. Los edificios se ubicaron manteniendo los árboles existentes, lo que no había autorizado antes permitió el uso de los tradicionales proyectos típicos en forma de bloques prismáticos. En este caso se tipificaron módulos habitacionales, por ejemplo, de dos viviendas conectadas por una circulación vertical, que podían combinarse de diferentes maneras aumentando la variedad de las soluciones arquitectónicas y su adecuación al contexto, y reduciendo así, la monotonía del conjunto urbano con respecto a aquellos que se habían desarrollado a partir de la repetición de un único modelo de edificio típico.

La variación en la altura de los edificios y el uso de una amplia gama de elementos constructivos y de terminación que recuperaron la tradición artesanal, también contribuyeron a la buena calidad de los resultados. Los espacios exteriores directamente relacionados con las viviendas en planta baja se asignaron a éstas como jardines en el frente o patios al fondo, lo cual contribuyó a mejorar la escala, el ambiente y el mantenimiento de las áreas verdes con respecto a los anteriores desarrollos urbanos abiertos. Esta práctica había sido abandonada desde el proyecto de la Unidad Vecinal Camilo Cienfuegos en La Habana del Este al inicio de los '60s.

Otro importante proyecto de vivienda urbana desarrollado durante los '80s y construido entre 1990 y 1991 fue la "Villa Panamericana" (ejecutada para alojar a los deportistas que participaron en los Juegos Panamericanos celebrados en La Habana en ese año), que recuperó algunos valores reconocidos de la ciudad tradicional, tales como su estructura en manzanas (super-manzanas en este caso), donde se insertaron edificios no típicos, proyectados por diferentes arquitectos, siguiendo las regulaciones establecidas en el Plan General Urbano (Figuras 3 y Foto 14).

Este método de proyecto garantizó la armonía y variedad del ambiente urbano, en el cual se mantiene la continuidad de la línea de fachada y los espacios verdes abiertos se sitúan hacia el interior de la manzana. La calidad del diseño arquitectónico no es uniforme, ya que algunos arquitectos no pudieron evitar la influencia del estilo post-moderno tardío, pero la calidad general del ambiente urbano es indudablemente mejor que en las anteriores urbanizaciones de vivienda. Las plantas bajas de los edificios se aprovecharon en muchos casos como espacios de parqueo, y en ellas se localizaron ser- 


\section{VILLA PANAMERICANA, MAQUETA Y PLAN GENERAL, 1991.}

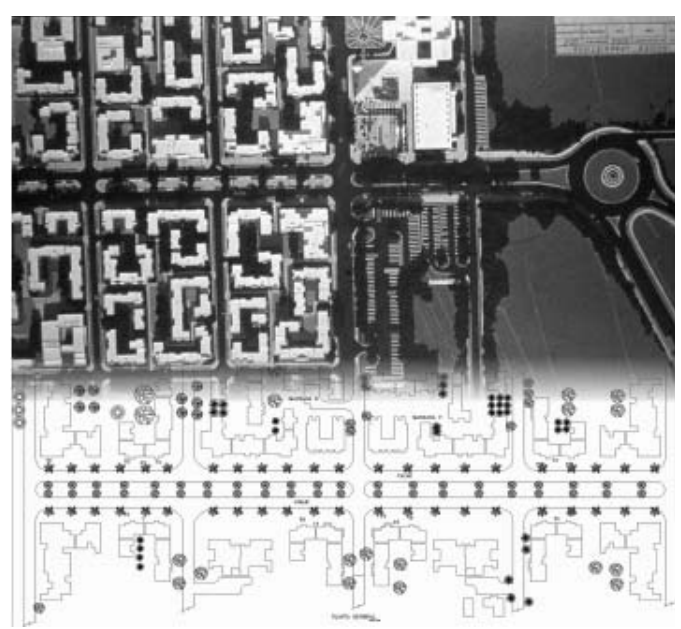

FIGURA 3

Foto suministrada por la Empresa de Proyectos DCH.

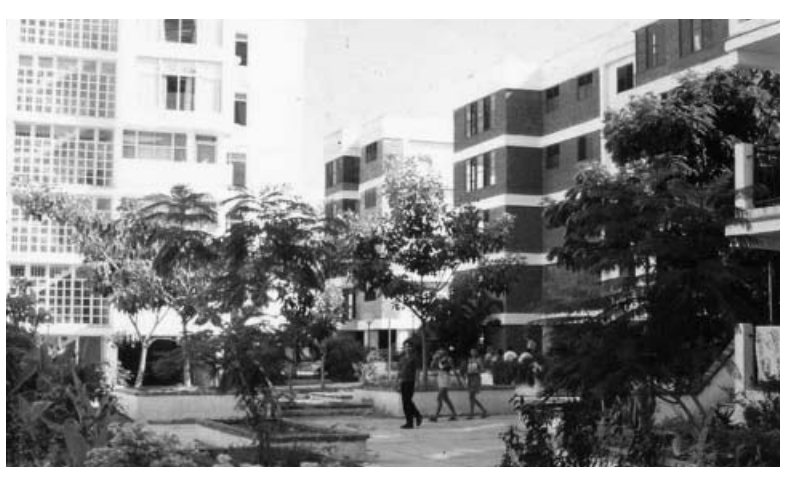

FOTO 14: Villa Panamericana, 1991.

Foto suministrada por la Empresa de Proyectos DCH. vicios comerciales hacia la avenida principal, donde lamentablemente nunca llegó a ejecutarse el clásico portal o corredor urbano que caracteriza las principales vías comerciales de la ciudad y que permite a las personas recorrer grandes distancias protegidas del sol y la lluvia.

\section{Los programas priorizados de viviendas}

Algunos programas de vivienda comenzaron a desarrollarse a finales de los '80s como apoyo a actividades económicas y sociales priorizadas. Tal es el caso del "Plan Turquino" para el desarrollo de las regiones montañosas que habían ido quedando despobladas como consecuencia de la emigración de la población joven hacia los llanos y los núcleos urbanos; el "Programa Alimentario" para el desarrollo de nuevas comunidades agrícolas que permitieran rescatar la población y, por tanto, la fuerza de trabajo en los asentamientos rurales que rodean la ciudad de La Habana y que también había ido emigrando; los "Polos Científicos", donde habitarían los trabajadores de los nuevos centros de investigación que se estaban creando en el país, pero sobre todo en la ciudad de La Habana y cuya producción constituye hoy una importante fuente de ingresos, y los "Polos Turísticos" que generaban asentamientos para los trabajadores de este relevante sector de la economía nacional. 
Como que se trataba en todos los casos de comunidades y viviendas para familias que laboraban en sectores priorizados de la economía, cuya fuerza de trabajo debía ser retenida, las soluciones debían resultar muy atractivas. Se aprobaron entonces regulaciones (instrucciones técnicas) especiales para estos tipos de vivienda que favorecían una mayor calidad con respecto a la vivienda social normada hasta entonces de manera general para toda la población cubana.

La crisis económica de los '90s interrumpió el desarrollo de tales programas en la forma en que éstos habían sido originalmente concebidos, por tanto, las realizaciones prácticas fueron en algunos casos escasas e incompletas y los enfoques tuvieron que ser cambiados para adaptarse a las nuevas y reales condiciones económicas.

\section{La crisis de los '90s. Nuevas vías "alternativas"}

Después de la caída del muro de Berlín fue muy difícil para la Revolución cubana sobrevivir, preservando los logros sociales alcanzados. Las relaciones comerciales establecidas y desarrolladas durante cuatro décadas con los países de Europa del Este se vieron cortadas de repente y Cuba no tenía mercados donde comprar ni vender sus productos. La escasez de muchos recursos esenciales tales como la energía y los alimentos impusieron la necesidad de detener o posponer algunos programas sociales e incluso económicos.

En tales condiciones, no era posible continuar la construcción industrializada. Para evitar detener totalmente la construcción de viviendas fue necesario reducir el consumo por unidad de diversos recursos escasos, tales como cemento, acero, madera, cables eléctricos e instalaciones hidrosanitarias. Como respuesta a esto nació en 1992 el llamado "Movimiento de viviendas de bajo consumo material y energético" y grandes esfuerzos se encaminaron hacia la búsqueda de tecnologías "alternativas" de construcción para hacer posible este objetivo (Foto 15).

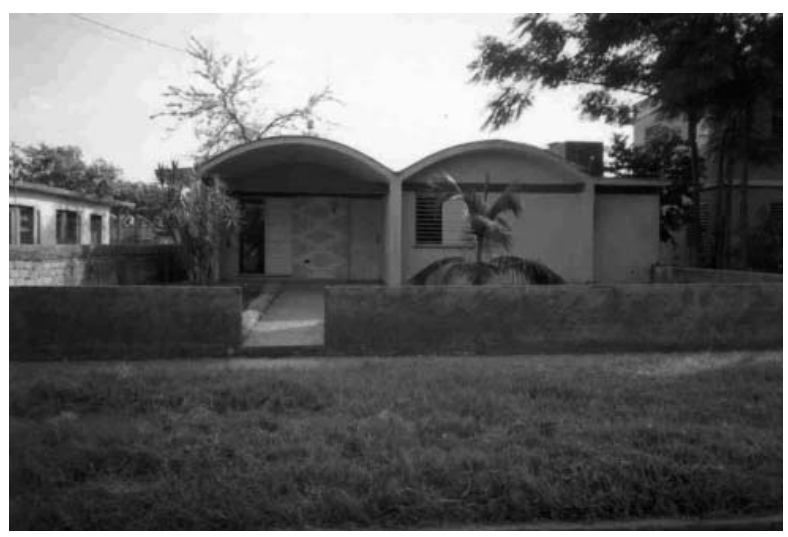

FOTO 15: Primera vivienda construida con bloques machihembrados de suelo cemento en la ciudad de Matanzas (proyectada por la autora en 1992). Esta fue la solución constructiva más empleada en las viviendas de "bajo consumo" a lo largo del país durante la década de los años '90s. Foto tomada por la autora, 1993. 
A pesar de que el espíritu y los objetivos de este movimiento eran justos, sus resultados no fueron tan económicos como se pretendía. Por el contrario, no solo la calidad de las viviendas decreció considerablemente, sino también su durabilidad se redujo por la baja calidad de los materiales, elementos de construcción, ejecución y terminación, y también porque la forma arquitectónica, en muchos casos, no se relacionaba ni con las nuevas tecnologías ni con las condiciones climáticas.

Adicionalmente, la tipología arquitectónica empleada se basaba fundamentalmente en unidades aisladas de una planta que hacían un uso extensivo del suelo urbano y por tanto, generaban altos costos de infraestructura. Finalmente, la durabilidad fue reducida, mientras que los costos de mantenimiento y explotación (consumo de energía a lo largo de la vida útil de la vivienda) y también los costos del suelo y la infraestructura se incrementaron ${ }^{10}$.

\section{Vivienda autoconstruida 0 "por medios propios"}

La auto-construcción, ya sea para ejecutar nuevas viviendas o para conservar las existentes, ha sido una alternativa siempre desarrollada de forma paralela desde 1959. Sin embargo, al contrario de lo que sucede en la mayoría de los países, quienes construyen por medios propios en Cuba no son los que no pueden acceder al mercado formal de la vivienda (que no existe en Cuba), sino aquellos que tienen dinero suficiente para afrontar sus necesidades habitacionales por sí mismos, sin ninguna ayuda.

Durante los años '80s, cuando la autoconstrucción se vio estimulada por la legislación vigente, la población, por sí misma, construyó más viviendas que el Estado, lo cual confirma su enorme potencial. No obstante, la principal dificultad que ha conspirado contra esta modalidad en Cuba ha sido siempre el limitado mercado de materiales y elementos de construcción para la venta a las familias que construyen por su cuenta. Por otra parte, su mayor debilidad consiste en el bajo aprovechamiento que genera el modelo de la vivienda unifamiliar aislada mientras el proceso de gestión se desarrolle de forma individual y las familias que autoconstruyen no se agrupen de alguna manera, por ejemplo, en cooperativas.

La participación de los profesionales en el proceso de auto-construcción fue limitada o algo "formal" antes de la creación del movimiento de los "Arquitectos de la Comunidad" en los "90s, de manera que la escasa participación de los arquitectos en las viviendas construidas por la población influyó en su baja calidad de diseño. Por otro lado, el modelo

10 González, Dania, 1997. 
de diseño seguido por la población como máxima aspiración se correspondía con la casa suburbana de la clase media de los '50s, la cual era, a su vez, una mala copia del diseño moderno de alta calidad de las residencias de las clases altas. Sin embargo, recientemente se ha estado produciendo una fuerte y generalizada tendencia al empleo de elementos ornamentales de corte clásico, tal vez como reacción a la desnudez de la arquitectura moderna que ha predominado durante los últimos 50 años (Foto 16).

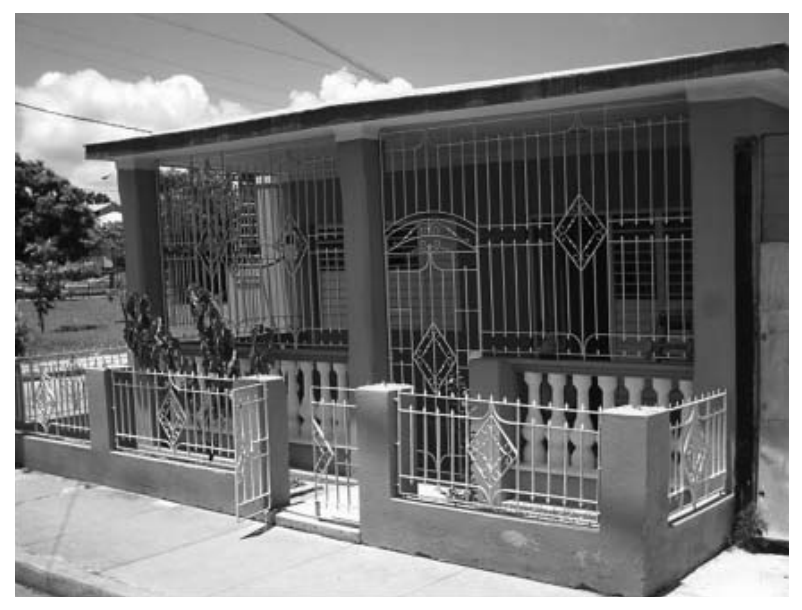

FOTO 16: Vivienda autoconstruida por "esfuerzo propio" en Holguín. Foto tomada por la autora, julio 2007.

El "Arquitecto de la Comunidad" comenzó trabajando con la población en forma participativa, apli- cando el "Método" desarrollado por el arquitecto argentino Rodolfo Livingston ${ }^{11}$. Hoy ellos se encargan también de asesorar y ayudar a la población en asuntos legales de la vivienda.

\section{La última década}

La primera década del siglo XXI ha estado marcada hasta hoy por una relativa tendencia a la recuperación económica del país, a pesar de lo cual no ha sido posible volver totalmente al modelo de construcción industrializada de la vivienda social masiva a partir de proyectos repetitivos, ya que durante la crisis económica de la década anterior se perdió gran parte de la infraestructura industrial instalada.

Una de las vías de solución más importantes desarrolladas en Cuba durante el último decenio ha sido el llamado "Movimiento Popular", originado en la provincia de Las Tunas para la ejecución de viviendas con mampuesto por parte de la población, y que se ha querido extender a todo el país para involucrar a la población más que nunca antes en el proceso de producción del hábitat, a partir del reconocimiento de que ésta representa un importante potencial productivo y que el estado por sí solo no puede proporcionar a cada familia una vivienda digna.

11 Livingston, Rodolfo, 1995. 
Otra variante de aplicación del mismo principio ha sido la asignación a los trabajadores a través de los sindicatos, del derecho a construir una vivienda, para lo cual su centro de trabajo y el organismo al cual éste pertenece, los apoyan, facilitándoles la adquisición y traslado de los materiales de construcción. El resultado ha sido la producción de urbanizaciones de viviendas aisladas de una planta, con muy baja densidad, que no resultan apropiadas en todos los casos. Este mismo esquema de gestión y mecanismo de selección se emplea para el mantenimiento, reparación y rehabilitación del fondo habitacional existente.

Estas iniciativas se complementan con regulaciones que limitan la autoconstrucción o "esfuerzo propio" (que es como se denomina esta modalidad en Cuba) a familias que hayan sido seleccionadas por la comunidad o por sus centros de trabajo, de manera que la vivienda continúe siendo un bien social a cuyo acceso tengan prioridad los ciudadanos por sus méritos personales y no por su poder adquisitivo, es decir, por sus ingresos y la disponibilidad de recursos. Este sigue siendo, por tanto, un principio distintivo de la vivienda en Cuba: que es un bien social, un derecho de todos y que no existe una dependencia entre ésta y el ingreso familiar.

Por otro lado y de forma paralela, se sigue produciendo la vivienda gestionada y financiada por los organismos del estado, en los últimos años más dirigida por los centros de trabajo, los organismos y los ministerios con vistas a retener su fuerza de trabajo, pero ya no mediante la fórmula de la $\mathrm{Mi}$ crobrigada, sino de brigadas de construcción especializadas y con el empleo de nuevas tecnologías de construcción que están siendo introducidas en el país (Foto 17). Tal es el caso de los sistemas de moldes metálicos recuperables o aquellos que emplean elementos a base de poliestireno expandido en diversas modalidades.

También siguen existiendo los programas estatales dirigidos a sectores priorizados como son en este momento los médicos internacionalistas que laboran en diversos países de América Latina y África (Foto 18). Sin embargo, el mayor problema que esta vivienda estatal genera es la producción de zonas residenciales, que continúan siendo periféricas, habitadas por sectores poblacionales homogéneos, contrariamente a lo que una urbanización sustentable demanda. A esto se suma la rigidez y falta de calidad de los proyectos, que se subordinan a los requerimientos del uso más económico de las tecnologías. Las valoraciones y la toma de decisiones continúan siendo eminentemente tecnológicas y carecen de un enfoque integral.

El proceso de rehabilitación urbana no ha avanzado a la velocidad requerida, de manera que al menos en la ciudad de La Habana se han ido perdiendo edificaciones que no han podido ser recuperadas a tiempo. Tampoco la producción de nuevas viviendas ha logrado satisfacer el déficit, lo que ha 


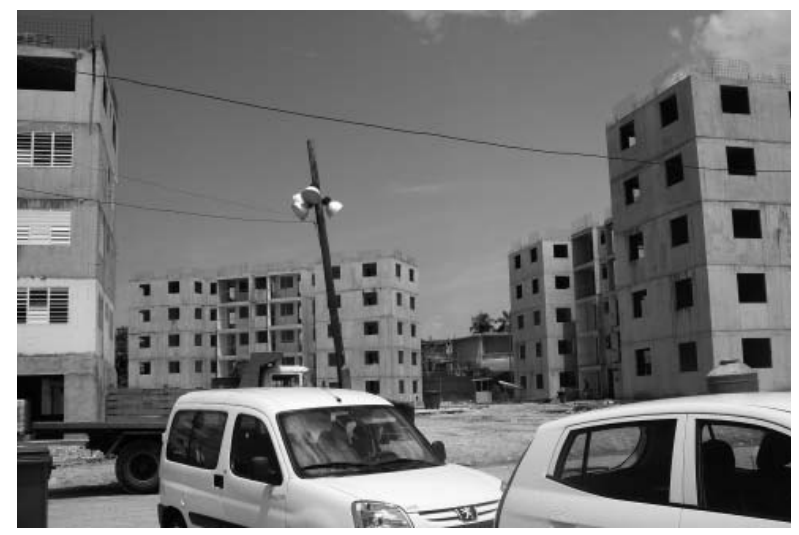

FOTO 17: Nuevas urbanizaciones que se ejecutan en la ciudad de La Habana con tecnología de sistema de moldes metálicos recuperables. Foto tomada por la autora en agosto 2008

provocado que numerosas familias se encuentren residiendo en albergues temporales en espera de alguna solución habitacional definitiva. Como una vía para resolver de forma emergente el déficit creciente, que se agrava en la ciudad capital, también se están produciendo actualmente cambios de uso en edificios de producción y servicios que se encuentran abandonados. La subdivisión de estos espacios de grandes dimensiones no siempre permite el logro de una adecuada relación de los espacios habitables interiores con el exterior para garantizar en ellos un ambiente térmico y visual adecuado.

Por otra parte, en aras de satisfacer las necesidades de crecimiento familiar y por tanto la demanda de

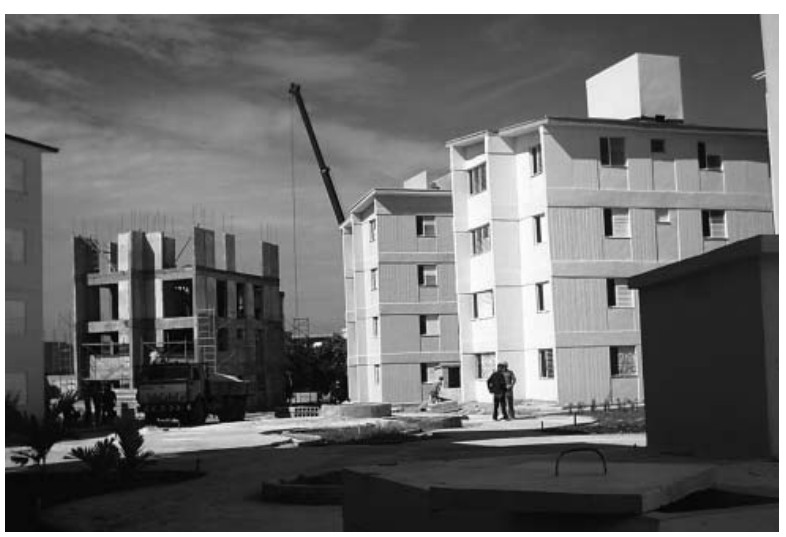

FOTO 18: Nueva urbanización en Holguín para viviendas de médicos. Foto tomada por la autora en octubre 2007.

nuevos espacios o por carecer de los recursos adecuados para la conservación de las viviendas, las familias que habitan en zonas centrales urbanas, incluso aquellas de reconocidos valores, realizan intervenciones constructivas y transformaciones que si bien van encaminadas a la solución parcial de sus necesidades inmediatas, provocan un alto impacto urbano mediante la alteración de la fachada de edificaciones de valor o la violación de las regulaciones urbanas, incluyendo la apropiación del espacio público o semipúblico. El debate sobre la connotación cultural de la arquitectura y el urbanismo ha comenzando. Sin embargo, esto aún no ha sido suficientemente interiorizado por los decidores ni por la propia población. 


\section{Reflexiones finales}

El ideal de una nueva vivienda que expresara en su forma y tecnología los nuevos contenidos de una sociedad más justa en un país pobre no ha logrado materializarse, quizás por las influencias externas que han conspirado en contra de la búsqueda de reales soluciones endógenas.

Tal vez éste sea el momento de recuperar las teorías de los años '60s, adaptadas a las nuevas circunstancias y a la experiencia de medio siglo, aprovechando las potencialidades, a partir de un enfoque integral sustentable, en el siglo XXI.

\section{Bibliografía}

ALFONSO, ALFONSO; DÍAZ,GISELA, Y DE LA PEÑA, ANA MARÍA. Por el rescate de la tradición. En Revista Arquitectura y Urbanismo. Mayo - agosto de 1989. Vol. X, № 2, págs. $2-7$.

ARMAS, RENDEL, Y MORALES, BORIS. Estudio de modelos urbanos para zonas residenciales en Ciudad de La Habana. Trabajo de Diploma, Facultad de Arquitectura, ISPJAE. La Habana. 2000. Tutor: Dra. Arq. Dania González.

CASTRO, FIDEL. La Historia me absolverá. $2^{a}$. ed. La Habana, Editorial Política. 1970.60p.

CUBA. Architecture in Countries in the Process of Development. Seventh Congress if the International
Union of Architects, La Habana. Septiembre de 1963. 118p.

GONZÁLEZ, DANIA. Hábitat rural: pasado, presente y futuro. En Revista Arquitectura y Urbanismo. Mayo - agosto de 1993. Vol. XIV, No 2, págs. 51 -60 .

GONZÁLEZ, DANIA. Economía y calidad en la vivienda. Un enfoque cubano. $1^{\text {a }}$. ed. La Habana, Editorial Científico Técnica. 1997. 167p.

GONZÁLEZ, DANIA. Del Reino de la Necesidad al Reino de la Abundancia y la Cultura. Merecido homenaje a mi tutor. En Revista Arquitectura y Urbanismo. Septiembre - diciembre de 2002. Vol. XXIII, No 3, págs. 32 - 35.

LIVINGSTON, RODOLFO. El Método. 1a. ed. Caracas, Ediciones de la Urraca. 1995. 200p.

REBELLÓN, JOSEFINA. Arquitectura y desarrollo nacional. Cuba 1978. $1^{a}$. ed. La Habana, Editorial CEDITEC. 1978. 230p.

SEGRE, ROBERTO. 10 años de Arquitectura en Cuba Revolucionaria. 1ª ed. La Habana, Ediciones Revolucionarias. 1970. 150p.

VEJAR, CARLOS. Y el perro ladra y la luna enfría. Fernando Salinas: diseño, ambiente y esperanza. $1^{\mathrm{a}}$. ed. La Habana, Ediciones Unión. 1994. 312p. 